\title{
IMPROVEMENT OF LTE DOWNLINK SYSTEM Performances Using THE LAgRange POLYNOMIAL INTERPOLATION
}

\author{
Mallouki Nasreddine, Nsiri Bechir, Walid Hakimi and Mahmoud Ammar \\ University of Tunis El Manar, National Engineering School of Tunis, LR99ES21 Lab. \\ Sys' Com, ENIT Tunis, Tunisia
}

\begin{abstract}
To achieve a high speed data rate, higher spectral efficiency, improved services and low latency the 3rd generation partnership project designed LTE standard (Long Term Evolution). the LTE system employs specific technical as well the technical HARQ, MIMO transmission, OFDM Access or estimation technical. In this paper we focus our study on downlink LTE channel estimation and specially the interpolation which is the basis of the estimation of the channel coefficients. Thus, we propose an adaptive method for polynomial interpolation based on Lagrange polynomial. We perform the Downlink LTE system MIMO transmission then compare the obtained results with linear, Sinus Cardinal and polynomial Newton Interpolations. The simulation results show that the Lagrange method outperforms system performance in term of Block Error Rate (BLER), throughput and EVN(\%)vs. Signal to Noise Ratio (SNR).
\end{abstract}

\section{KEYWORDS}

LTE; MIMO ;OFDM;EVM; Interpolation; Lagrange;

\section{INTRODUCTION}

In modern world, requirement of high data rate communication has become inevitable. Applications such as streaming transmission, video images, and World Wide Web browsing require high speed data transmission with mobility. In order to fulfill these data requirements, the 3rd Generation Partnership Project (3GPP) [1][2][3] introduced Long Term Evolution (LTE), to provide high speed data rate for mobile communication. The LTE system affords an important effective bit rate and allows increasing system capacity in terms of numbers of simultaneous calls per cell. In addition, it has a low latency compared to $3 \mathrm{G} / 3 \mathrm{G}+$ networks. It offers a theoretical speed of 100 Mbits / s in the Downlink and 50Mbits/s in the Uplink transmission. The LTE uses Orthogonal Frequency Division Modulation (OFDM) and Orthogonal Frequency Division Modulation multiple access technique (OFDMA) in the downlink transmission [4]. The OFDM provides the signal transmitted robustness against the multipath effect and can improve the spectral efficiency of the system [5][6]. On the other hand, the implementation of MIMO system increases channel capacity and decreases the signal fading by sending the same information at the same time through multiple antennas [6]. The combination of these two powerful technologies (MIMO-OFDM) in the LTE system improving thus the spectral efficiency and throughput offered without increasing resources for base bands and power output.

To best exploit the power of MIMO-OFDM technology, it is imperative to manage at best the estimation of the channel coefficients; this operation is ensured by the interpolation of pilots. 
In this paper, we represent a polynomial interpolation algorithm using the method of Lagrange [12] which greatly reduces the complexity of the transceiver. The simulation is made on a 'Vehicular A' (Veh A) [13].channel through MIMO system using Least Square equalizer (LS). Section II of this paper give an over view of MIMO-OFDM transmission. In Section III, we present Lagrange interpolation algorithm. Finally, Section IV provides the numerical results.

\section{MIMO-OFDM TRANSMISSION}

\subsection{MIMO OFDM transmissions schemes [3]}

In this section, we describe the MIMO OFDM transceiver. A modulation block is used to modulate the original binary data symbol using the complex constellation QPSK, 16 QAM or 64 QAM according to the LTE standard [8][9]. Pilot insertion is generated according to the LTE standards, followed by Inverse Fast Fourier Transform operation (IFFT); at the end, a cyclical prefix is inserted to remedy the phenomenon of the Inter Symbol Interference (ISI) and the Inter Sub carriers Interference. Transmission is made through a multipath Fast Fading channel over a multiple antenna system. Multiple antennas can be used in the transmitter and the receiver; consequently, MIMO encoders are needed to increase the spatial diversity or the channel capacity. Applying MIMO allows us to get a diversity gain to remove signal fading or getting a gain in terms of capacity. Generally, there are three types of MIMO receivers, as presented in [1]. At the reception, the cyclical prefix is firstly removed, followed by the Fast Fourier Transform operation (FFT); after the extraction of pilots, parameters of channel is estimated through the block interpolation followed by equalization. The method of interpolation chosen is essential to make the estimation more efficient and to reduce the equalizer complexity.

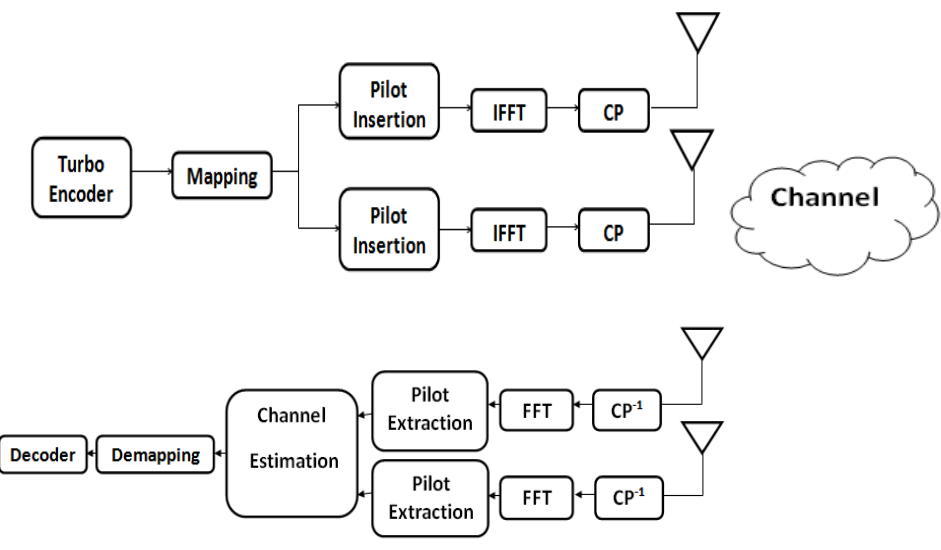

Figure 1. MIMO-OFDM transmission

\subsection{Analysis of standard LTE pilot scattering}

In the LTE standards, pilots are placed in a well-defined ways to cover up the frequency and time domain. The location of pilots for 2x2 MIMO transmissions scheme in LTE system is shown in the following figures. 


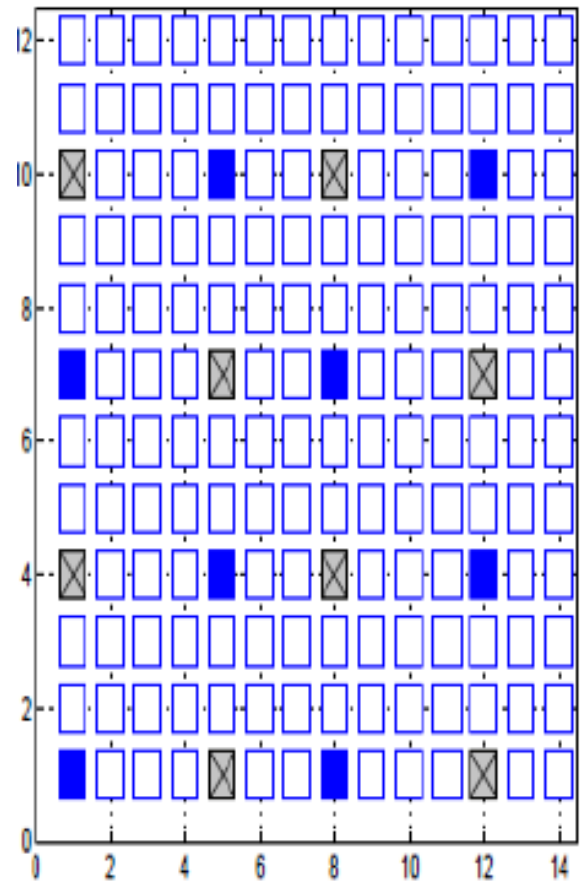

Time Symbol index

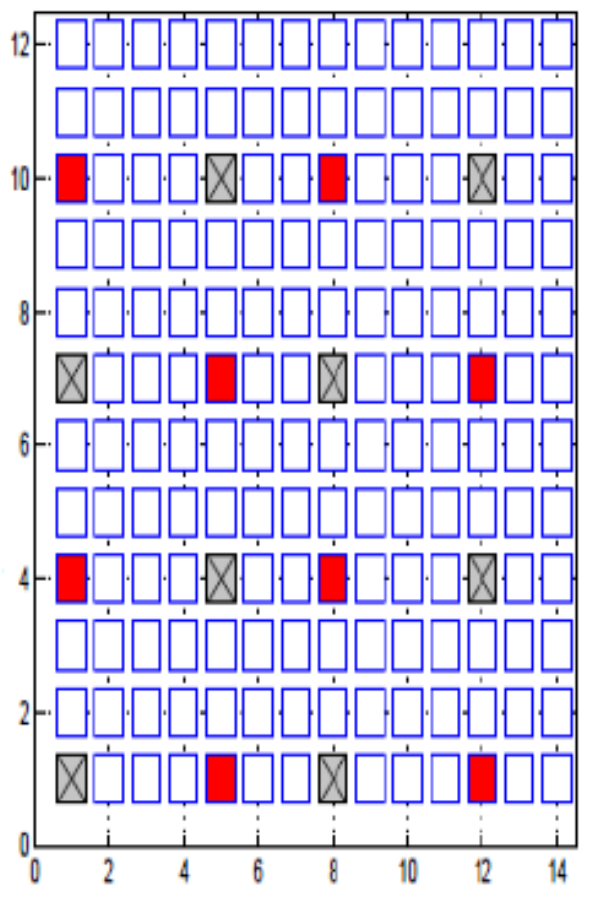

Time Symbol index

Figure 2. Pilot structure of Transmitter $1 \& 2$

It can be seen that, through the first antenna, pilots are disposed in OFDM symbols numbers 1, 5, 8 and 12 while for the second antenna, they are placed in the same OFDM symbols, but in different subcarriers index. Those positions allow a better coverage of the frequency and time and reduce the risk of interference in reception [4].

\subsection{Error Vector Magnitude (EVM)[7]}

Error vector magnitude (EVM) is a measure of modulation quality and error performance in complex wireless systems. It provides a method to evaluate the performance of software-defined radios (SDRs), both transmitters and receivers. It also is widely used as an alternative to bit error rate (BER) measurements to determine impairments that affect signal reliability. (BER is the percentage of bit errors that occur for a given number of bits transmitted.) EVM provides an improved picture of the modulation quality as well.

EVM measurements are normally used with multi-symbol modulation methods like multi-level phase-shift keying (M-PSK), quadrature phase-shift keying (QPSK), and multi-level quadrature amplitude modulation (M-QAM). These methods are widely used in wireless local-area networks (WLANs), broadband wireless, and 4G cellular radio systems like Long-Term Evolution (LTE) where M-QAM is combined with orthogonal frequency division multiplexing (OFDM) modulation.

EVM is the ratio of the average of the error vector power (Perror) to the average ideal reference vector power (Pref) expressed in decibels. The averages are taken over multiple symbol periods: 
International Journal of Computer Networks \& Communications (IJCNC) Vol.6, No.5, September 2014

$$
\operatorname{EVM}(d B)=10 \log (\text { Perror } / \operatorname{Pr} \text { ef })
$$

You will also see it expressed as a percentage:

$$
\operatorname{EVM}(\%)=\sqrt{\text { Perro } / \operatorname{Pr} e f} * 100
$$

\section{DESCRIPTION OF THE INTERPOLATION ALGORITHM}

\subsection{Linear Interpolation}

In linear polynomial interpolation, the channel coefficients are estimated using the linear relationship between two successive pilots.

Linear interpolation is given by the following expression:

$$
H_{(k)}^{(i)}=(i / d) * H_{(k)(p)}^{(i)}+(1-(i / d)) * H_{(p+1)}^{(i)}
$$

where $H_{(k)}^{(i)}$ is the channel coefficient to estimate, $H_{(k)(p)}^{(i)}$ and $H_{(p+1)}^{(i)}$ two successive pilots, i is the subcarriers index, $\mathrm{k}$ is the OFDM symbol index, $\mathrm{p}$ is the pilot index and $\mathrm{d}$ is the distance between two pilots [10].

\subsection{Sinus Cardinal Interpolation}

Sinus Cardinal (SinC) interpolation is given by the following expression [11]:

$$
S(x)=\sum_{i=0}^{n} S(k) \sin c(x-k)
$$

Where $S(k)$ are the pilots, $\mathrm{k}$ is the position of $\mathrm{y}, S(x)$ is the Sinus Cardinal interpolation function. In this work, we use 2 pilots to estimate channel coefficients using Sinus Cardinal Interpolation. The interpolation is represented as follow:

1-Extract received $y_{(k)(p)}^{(i)}$ pilots from received signal $y_{(k)}^{(i)}$

2-Calculate the channel coefficients of pilots symbols with Least Square estimator

$$
H_{(k)(p)}^{(i)}=y_{(k)(p)}^{(i)} / x_{(k)(p)}^{(i)}
$$

3- Estimate $H_{(k)}^{(i)}$ with Sinus Cardinal interpolation:

$$
H_{(k)}^{(i)}=\sin c\left(x-x_{P 0}\right) * H_{(k)(P 0)}^{(i)}+\sin c\left(x-x_{P 1}\right) * H_{(k)(P 1)}^{(i)}
$$

\subsection{Newton polynomial Interpolation:}

Newton polynomial Interpolation is given by the following expression [12]: 
International Journal of Computer Networks \& Communications (IJCNC) Vol.6, No.5, September 2014

$$
\begin{gathered}
P_{n}(x)=\sum_{i=0}^{n} a_{i} N_{i}(x) \\
N_{i}(x)=\prod_{j=0}^{i-1}\left(x-x_{j}\right) \\
a_{i}=f\left[x_{0} \ldots \ldots \ldots \ldots . . . . . x_{i}\right] \\
f\left[x_{0}, x_{1}\right]=\left(f\left[x_{0}\right]-f\left[x_{1}\right]\right) /\left(x_{0}-x_{1}\right)
\end{gathered}
$$

$a_{0} \ldots \ldots . a_{n}$ are the coefficients of Newton polynomial of order $\mathrm{n}, P_{n}$ is the polynomial of Newton and $x_{i}$ are the pilots frequency indexes.

Estimate $H_{(k)}^{(i)}$ with Newton polynomial:

$$
H_{(k)}^{(i)}=P_{n}(x)=\sum_{i=0}^{n} a_{i} N_{i}(x)
$$

\subsection{Lagrange polynomial Interpolation}

Lagrange polynomial allows interpolating a set of points by a polynomial which goes exactly through these points. The Lagrange polynomial is given by the following expression [12]

$$
\begin{gathered}
P(x)=\sum_{i=0}^{n} y_{i} L_{i}(x) \\
L(x)=\prod_{\substack{j=0 \\
j \neq i}}^{n}\left(x-x_{i}\right) /\left(x_{i}-x_{j}\right)
\end{gathered}
$$

Where $y_{i}$ the pilots, $\boldsymbol{x}$ is the position of $y, L$ is the coefficients of Lagrange and $n$ is the Lagrange polynomial order.

\subsection{Algorithm description}

The received signal for MIMO system model consisting of $N_{T}$ transmits antennas and $N_{R}$ receives antennas can be represented by the following Equation:

$$
Y_{(k)}^{(i)}=X_{(k)}^{(i)} * H+N
$$

Where $Y_{(k)}^{(i)}=\left[y_{0}^{0} \ldots \ldots \ldots . . . y_{0}^{N_{S C}} \ldots \ldots \ldots \ldots . . . y_{N O F D M_{-} S Y M N_{R}}^{N_{S C}}\right]$ is the received vector, $H_{(k)}^{(i)}$ is the channel coefficient matrix of the dimensions $N_{T} \times N_{R}$ express the channel gain and $\mathrm{N}=[\mathrm{n} 1, \mathrm{n} 2$ $\ldots . . n N_{R}$ ] T is the noise vector. 
The matrix $H_{(k)}^{(i)}$ is written as follow [12][13]:

$$
H_{(k)}^{(i)}=\left(\begin{array}{l}
h_{(k)}^{(i)} h_{(k) 1,2}^{(i)} \ldots \ldots \ldots . . . h_{(k) 1, N_{T}}^{(i)} \\
h_{(k) 2,1}^{(i)} h_{(k) 2,2}^{(i)} \ldots \ldots \ldots \ldots . . . . . .(k) 2, N_{T} \\
\vdots \\
h_{(k) N_{R}, 1}^{(i)} h_{(k) N_{R}, 2}^{(i)} \ldots \ldots \ldots . . . h_{(k) N_{R}, N_{T}}^{(i)}
\end{array}\right)
$$

For each reception antennas, after eliminating Cyclical Prefix and Fast Fourier Transform operation, pilots are extracted and then interpolation block is attacked to estimate the parameter $H_{(k)}^{(i)}$ of the channel. The interpolation operation is necessary for both frequency and time domain. In the present work, we use a Lagrange polynomial interpolation for frequency domain and linear interpolation for temporary. The interpolation algorithm is represented in figure4.

The steps of algorithm are described as follow:

1-Extract $y_{(k)(p)}^{(i)}$ pilots from received signal $y_{(k)}^{(i)}$

2-Calculate the channel coefficients of pilots symbols with

Least Square estimator

$$
H_{(k)(p)}^{(i)}=y_{(k)(p)}^{(i)} / x_{(k)(p)}^{(i)}
$$

3-Calculate $L_{0} \ldots \ldots \ldots \ldots \ldots . L_{n}$ Coefficients of Lagrange with $\mathrm{n}$ order of Lagrange polynomial and $\mathrm{p}$ index of pilots, we start with $\mathrm{n}=2$. For example for 12 first coefficients to estimate we use 3 first pilots placed respectively at $x_{p 0}=0, x_{p 1}=6$ and $x_{p 2}=12$ frequency index

$$
\begin{aligned}
& L_{0}=\left(\left(x_{i}-x_{p 1}\right) *\left(x_{i}-x_{p 2}\right)\right) /\left(\left(x_{p 0}-x_{p 1}\right) *\left(x_{p 0}-x_{p 2}\right)\right) \\
& L_{1}=\left(\left(x_{i}-x_{p 0}\right) *\left(x_{i}-x_{p 2}\right)\right) /\left(\left(x_{p 1}-x_{p 0}\right) *\left(x_{p 1}-x_{p 2}\right)\right) \\
& L_{2}=\left(\left(x_{i}-x_{p 0}\right) *\left(x_{i}-x_{p 1}\right)\right) /\left(\left(x_{p 2}-x_{p 0}\right) *\left(x_{p 2}-x_{p 1}\right)\right)
\end{aligned}
$$

Where $L_{0}, L_{1}$ and $L_{2}$ are the coefficients of Lagrange polynomial of order $\mathrm{n}=3, x_{i}$ is the frequency index of $H_{(k)}^{(i)}$ to estimate and $x_{p 0}, x_{p 1}, x_{p 2}$ are the frequency indexes of first tree pilots. 


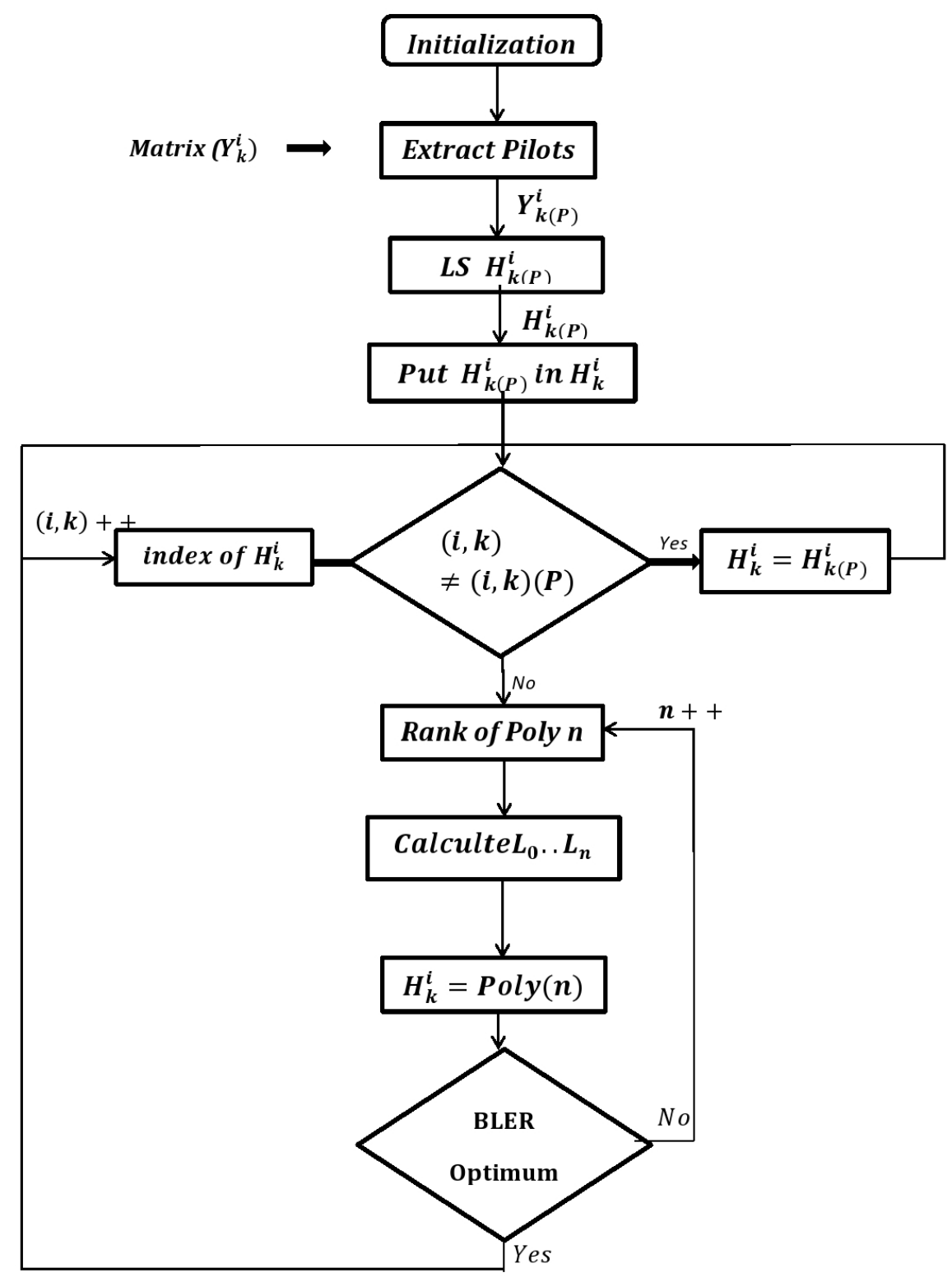

Figure 3. Algorithm of interpolation

4-Estimate $H_{(k)}^{(i)}$ with Lagrange polynomial:

$$
H_{(k)}^{(i)}=L_{0} * H_{(k)(p)}^{(i)}+L_{1} * H_{(k)(p+1)}^{(i)}+L_{2} * H_{(k)(p+2)}^{(i)}
$$

where $H_{(k)(p)}^{(i)}, H_{(k)(p+1)}^{(i)}$ and $H_{(k)(p+2)}^{(i)}$ are three successive pilots. 
International Journal of Computer Networks \& Communications (IJCNC) Vol.6, No.5, September 2014

5- Testing the estimation operation performance by incrementing the polynomial of order $\mathrm{n}$ until having optimal performance in term of Block Error Rate (BLER), Throughput and Error Vector Magnitude (EVM(\%)) vs. SNR.

\section{Simulations RESUlts}

Our simulations was performed for LTE downlink transmission through a channel which uses the profile of ITU-Veh A for MIMO system with use of 16 QAM (CQI=7) constellation. This simulation in divided in 2 parts, firstly we present BLER vs SNR over many values of the order of Lagrange Polynomial (n) to determinate the optimal one. In the second part, we showed simulation results for known channel, Lagrange polynomial interpolation algorithm, Sin Cardinal Interpolation, Newton polynomial interpolation and linear interpolation for optimal $\mathrm{n}$. All simulations are used over a Least Square equalizer. Simulation results are compared in term of Block Error Rate (BLER), Throughput and Error Vector Magnitude (EVM(\%)) vs. SNR. this System is simulated using the parameters shown in TABLE I [13][15].

TABLE 1. PARAMETERS SIMULATION.

\begin{tabular}{|l|l|}
\hline Transmission Bandwidth & $1.4 \mathrm{MHz}$ \\
\hline Carrier Frequency & $2.1 \mathrm{GHz}$ \\
\hline Data Modulation & $16 \mathrm{QAM}$ (CQI 7) \\
\hline Channel & ITU-Veh A \\
\hline Interpolation & $\begin{array}{l}\text { Polynomial interpolation OF LAGRANGE } \\
\text { Polynomial interpolation OF Newton } \\
\text { Sinus Cardinal Interpolation } \\
\text { Linear Interpolation }\end{array}$ \\
\hline
\end{tabular}

\subsection{Practical Determination of optimal n}

One of aim of our adaptive algorithm is to determinate an optimal value of $\mathrm{n}$ the order of Lagrange polynomial. In this part we show how our algorithm determinate n. For that, we choose 4 values of $\mathrm{n}$ and we present their performance in term of BLER vs SNR for a MIMO system.

- $\quad$ order of Polynomial $n=1$ (Linear interpolation)

$$
\begin{gathered}
L_{0}=\prod_{\substack{j=0 \\
j \neq i}}^{n}\left(\left(x-x_{p j}\right) /\left(x_{p 0}-x_{p j}\right)\right) \\
L_{1}=\prod_{\substack{j=0 \\
j \neq i}}^{n}\left(\left(x-x_{p j}\right) /\left(x_{p 1}-x_{p j}\right)\right) \\
H_{(k)}^{(i)}=L_{0} * H_{(k)(p 0)}^{(i)}+L_{1} * H_{(k)(p 1)}^{(i)}
\end{gathered}
$$

Where $L_{0}$ and $L_{1}$ are the coefficients of Lagrange polynomial of order $\mathrm{n}=1, x_{i}$ is the frequency index of $H_{(k)}^{(i)}$ to estimate, $x_{p j}$ are the frequency indexes of pilots and $H_{(k)(p 0)}^{(i)}, H_{(k)(p 1)}^{(i)}$ and two successive pilots. 
- $\quad$ order of Polynomial $n=3$ (Cubic interpolation)

$$
\begin{aligned}
L_{0} & =\prod_{\substack{j=0 \\
j \neq i}}^{n}\left(\left(x-x_{p j}\right) /\left(x_{p 0}-x_{p j}\right)\right) \\
L_{1} & =\prod_{\substack{j=0 \\
j \neq i}}^{n}\left(\left(x-x_{p j}\right) /\left(x_{p 1}-x_{p j}\right)\right) \\
L_{2} & =\prod_{\substack{j=0 \\
j \neq i}}^{n}\left(\left(x-x_{p j}\right) /\left(x_{p 2}-x_{p j}\right)\right) \\
L_{3} & =\prod_{\substack{j=0 \\
j \neq i}}^{n}\left(\left(x-x_{p j}\right) /\left(x_{p 3}-x_{p j}\right)\right) \\
H_{(k)}^{(i)}=L_{0} * H_{(k)(p 0)}^{(i)} & +L_{1} * H_{(k)(p 1)}^{(i)}+L_{2} * H_{(k)(p 2)}^{(i)}+L_{3} * H_{(k)(p 3)}^{(i)}
\end{aligned}
$$

Where $L_{0}, L_{1}, L_{2}$ and $L_{3}$ are the coefficients of Lagrange polynomial of order $\mathrm{n}=3, x_{i}$ is the frequency index of $H_{(k)}^{(i)}$ to estimate, $x_{p j}$ are the frequency indexes of pilots and $H_{(k)(p 0)}^{(i)}$, $H_{(k)(p 1)}^{(i)}, H_{(k)(p 2)}^{(i)}$ and $H_{(k)(p 3)}^{(i)}$ four successive pilots.

- $\quad$ order of Polynomial $n=5$

$$
\begin{aligned}
& L_{0}=\prod_{\substack{j=0 \\
j \neq i}}^{n}\left(\left(x-x_{p j}\right) /\left(x_{p 0}-x_{p j}\right)\right) \\
& L_{1}=\prod_{\substack{j=0 \\
j \neq i}}^{n}\left(\left(x-x_{p j}\right) /\left(x_{p 1}-x_{p j}\right)\right) \\
& L_{2}=\prod_{\substack{j=0 \\
j \neq i}}^{n}\left(\left(x-x_{p j}\right) /\left(x_{p 2}-x_{p j}\right)\right) \\
& L_{3}=\prod_{\substack{j=0 \\
j \neq i}}^{n}\left(\left(x-x_{p j}\right) /\left(x_{p 3}-x_{p j}\right)\right) \\
& L_{4}=\prod_{\substack{j=0 \\
j \neq i}}^{n}\left(\left(x-x_{p j}\right) /\left(x_{p 4}-x_{p j}\right)\right) \\
& L_{5}=\prod_{\substack{j=0 \\
j \neq i}}^{n}\left(\left(x-x_{p j}\right) /\left(x_{p 5}-x_{p j}\right)\right)
\end{aligned}
$$


International Journal of Computer Networks \& Communications (IJCNC) Vol.6, No.5, September 2014

$$
H_{(k)}^{(i)}=L_{0} * H_{(k)(p 0)}^{(i)}+L_{1} * H_{(k)(p 1)}^{(i)}+L_{2} * H_{(k)(p 2)}^{(i)}+L_{3} * H_{(k)(p 3)}^{(i)}+L_{4} * H_{(k)(p 4)}^{(i)}+L_{5} * H_{(k)(p 5)}^{(i)}
$$

Where $L_{0}, L_{1}, L_{2}, L_{3}, L_{4}$ and $L_{5}$ are the coefficients of Lagrange polynomial of order $\mathrm{n}=5, x_{i}$ is the frequency index of $H_{(k)}^{(i)}$ to estimate, $x_{p j}$ are the frequency indexes of pilots and $H_{(k)(p 0)}^{(i)}$ $, H_{(k)(p 1)}^{(i)}, H_{(k)(p 2)}^{(i)}, H_{(k)(p 3)}^{(i)}, H_{(k)(p 4)}^{(i)}$ and $H_{(k)(p 5)}^{(i)}$ four successive pilots.

- $\quad$ order of Polynomial $n=7$

$$
\begin{aligned}
& L_{0}=\prod_{\substack{j=0 \\
j \neq i}}^{n}\left(\left(x-x_{p j}\right) /\left(x_{p 0}-x_{p j}\right)\right) \\
& L_{1}=\prod_{\substack{j=0 \\
j \neq i}}^{n}\left(\left(x-x_{p j}\right) /\left(x_{p 1}-x_{p j}\right)\right) \\
& L_{2}=\prod_{\substack{j=0 \\
j \neq i}}^{n}\left(\left(x-x_{p j}\right) /\left(x_{p 2}-x_{p j}\right)\right) \\
& L_{3}=\prod_{\substack{j=0 \\
j \neq i}}^{n}\left(\left(x-x_{p j}\right) /\left(x_{p 3}-x_{p j}\right)\right) \\
& L_{4}=\prod_{\substack{j=0 \\
j \neq i}}^{n}\left(\left(x-x_{p j}\right) /\left(x_{p 4}-x_{p j}\right)\right) \\
& L_{5}=\prod_{\substack{j=0 \\
j \neq i}}^{n}\left(\left(x-x_{p j}\right) /\left(x_{p 5}-x_{p j}\right)\right) \\
& L_{6}=\prod_{\substack{j=0 \\
j \neq i}}^{n}\left(\left(x-x_{p j}\right) /\left(x_{p 6}-x_{p j}\right)\right) \\
& L_{7}=\prod_{\substack{j=0 \\
j \neq i}}^{n}\left(\left(x-x_{p j}\right) /\left(x_{p 7}-x_{p j}\right)\right) \\
& H_{(k)}^{(i)}=L_{0} * H_{(k)(p 0)}^{(i)}+L_{1}^{*} H_{(k)(p 1)}^{(i)}+L_{2} * H_{(k)(p 2)}^{(i)}+L_{3}^{*} H_{(k)(p 3)}^{(i)}+L_{4}^{*} H_{(k)(p 4)}^{(i)}+L_{5} * H_{(k)(p 5)}^{(i)}+L_{6}^{*} H_{(k)(p 6)}^{(i)}+L_{7} * H_{(k)(p 7)}^{(i)}
\end{aligned}
$$

Where $L_{0}, L_{1}, L_{2}, L_{3}, L_{4}, L_{5}, L_{6}$ and $L_{7}$ are the coefficients of Lagrange polynomial of order $\mathrm{n}=5, \quad x_{i}$ is the frequency index of $H_{(k)}^{(i)}$ to estimate, $x_{p j}$ are the frequency indexes of pilots and $H_{(k)(p 0)}^{(i)}, H_{(k)(p 1)}^{(i)}, \quad H_{(k)(p 2)}^{(i)}, H_{(k)(p 3)}^{(i)}, H_{(k)(p 4)}^{(i)}, H_{(k)(p 5)}^{(i)}, H_{(k)(p 6)}^{(i)}$ and $H_{(k)(p 7)}^{(i)}$ are eight successive pilots. 


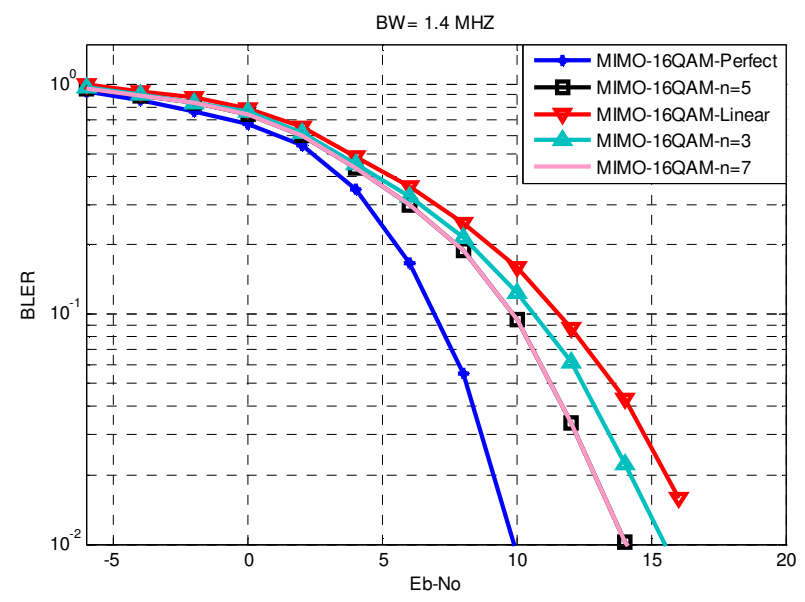

Figure 4. BLER vs. SNR for MIMO Transmission over Veh-A channel, CQI=7.

In Figure 4 the BLER vs SNR of LTE Downlink system over MIMO transmission for different value of the order of Lagrange Polynomial(n), is showed. We notice that the proposed algorithm of polynomial interpolation gives the best performances for $n=5$ which is considered as an optimal order of polynomial of interpolation. We also see, that for $n$ more than 5(in our case $n=7$ ) we have the same performance in term of BLER vs SNR for these conditions of transmission.

\subsection{Simulation results and discussion}

To observe the effect of the Lagrange polynomial interpolation compared to linear, Sin Cardinal and Newton interpolation techniques, we simulate and plot the performance of LTE Downlink system in MIMO transmission over multipath channel (ITU-Veh A) using an LS equalizer. The simulations have been carried out for the 16-QAM (CQI=7). The Block Error Rate (BLER), throughput and Error Vector Magnitude (\%) vs. SNR results were study. Figure 5 show Block Error Rate vs. SNR for known channel, Lagrange polynomial, Sinus Cardinal ,Newton polynomial and linear interpolations for MIMO transmission.

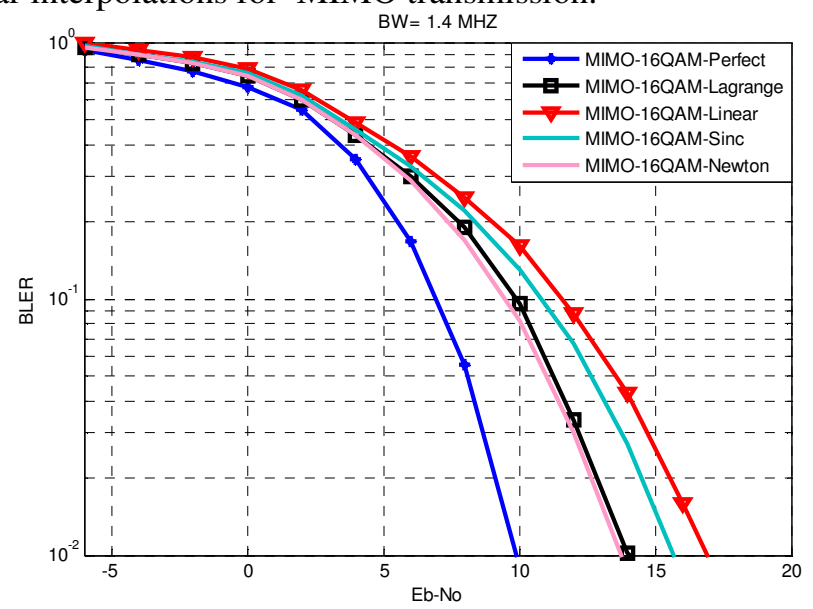

Figure 5. BLER vs. SNR for MIMO Transmission over Veh-A channel, CQI=7.

We can see that the proposed adaptive algorithm of Lagrange polynomial interpolation enhances the performance of downlink LTE system by almost than $2 \mathrm{~dB}$ for BLER $=10-1$ compared to the Linear and the Sinus Cardinal Interpolations. On the other hand, we also see that performance of 
MIMO system using Polynomial Lagrange and Polynomial Newton interpolation gives the same results in term of BLER vs SNR despite that newton interpolation is more complex.

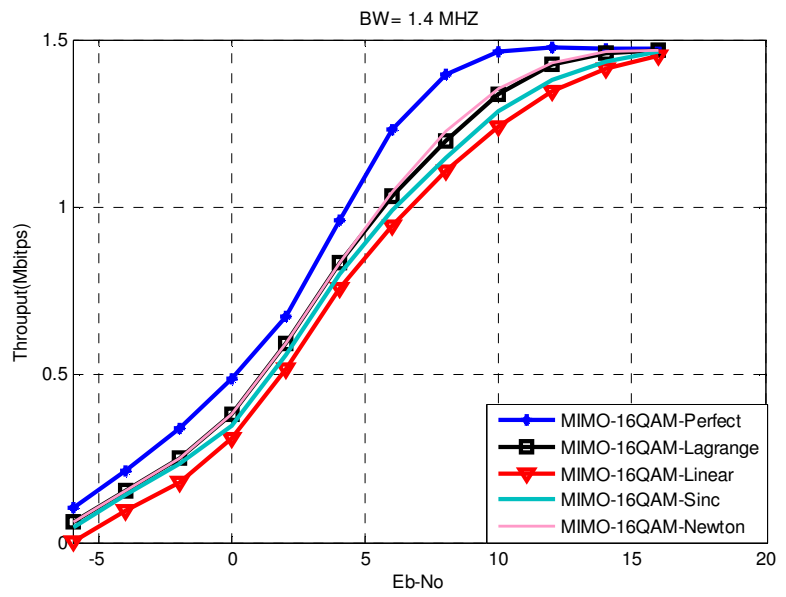

Figure 6. Throughput vs. SNR for MIMO Transmission over Veh-A channel, CQI=7.

As shown in Figure 6 the throughput of MIMO transmission for CQI=7 over Vehicular A channel for known channel, Lagrange polynomial, Sinus Cardinal ,Newton polynomial and linear interpolations are investigated. We can note that the suggested algorithm of polynomial interpolation improves the throughput compared to the linear and the Sinus Cardinal interpolation. For example, with throughput=1 MHz we have a gain almost than $1 \mathrm{~dB}$ for MIMO systems.

On the other side, performance of LTE Downlink system in term of Throughput vs SNR using Lagrange interpolation gives the same results compared to the Newton Polynomial interpolation

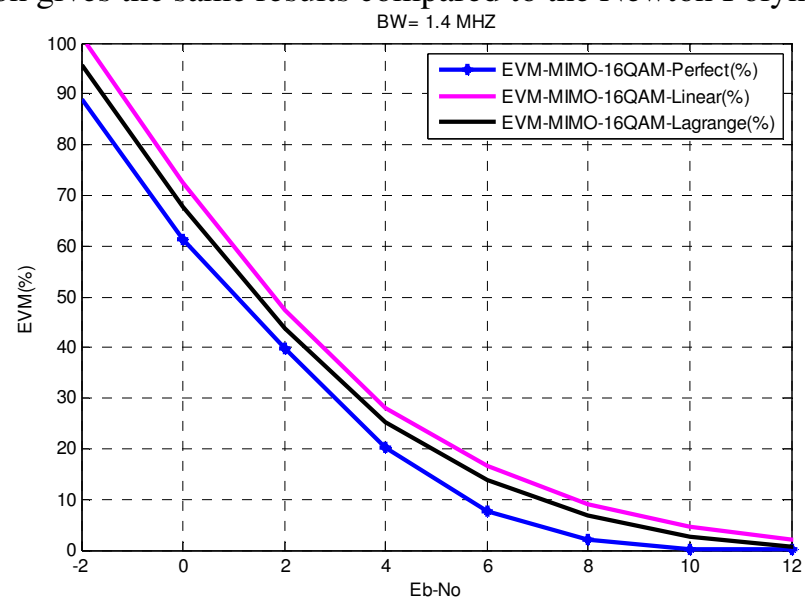

Figure 7. EVM(\%) vs. SNR for MIMO Transmission over Veh-A channel, CQI=7.

Figure 7 shows the Error Vector Magnitude vs SNR for LTE Downlink system using Known Channel, Linear interpolation and Lagrange polynomial interpolation. We note, that the Lagrange Polynomial interpolation enhances measured EVM (\%) compared to the Linear interpolation by almost $3 \%$ for a $\mathrm{SNR}=8 \mathrm{~dB}$. 


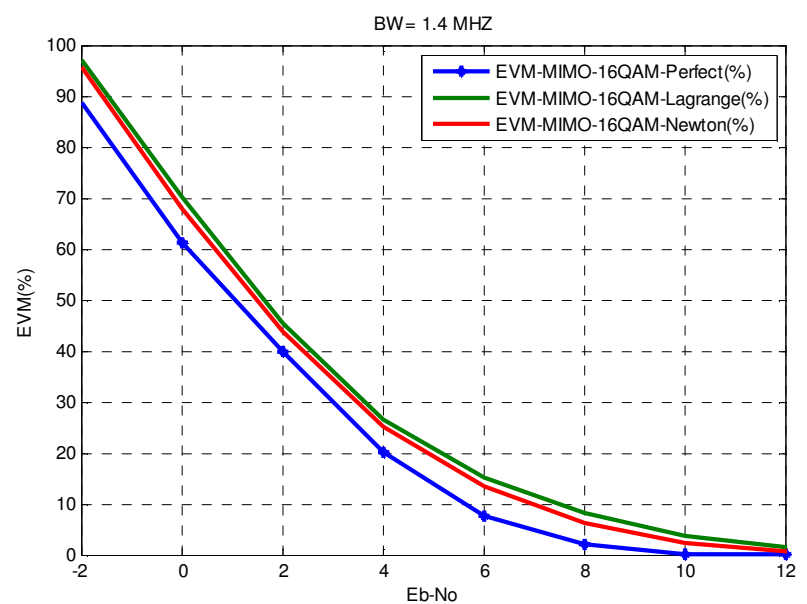

Figure 8. EVM(\%) vs. SNR for MIMO Transmission over Veh-A channel, CQI=7.

Measured EVM (\%) of Lagrange polynomial interpolation and Newton Polynomial interpolation are investigated in Figure 8, we find that, these two techniques of interpolation have got the same performance in term of EVM $(\%)$

After studying performance, we find that the Lagrange polynomial interpolation offers a significant improvement compared to the linear and the Sinus Cardinal Interpolations in term of BLER, Throughput and EVM(\%) vs SNR, as a result of the precision given by using $\mathrm{n}$ pilots to estimate each channel parameter. In fact, the use of $n$ pilots in estimation of the channel coefficients takes into account the correlation between the pilot subcarriers; which makes this calculation more accurate and thus enhances the system efficiency.

It is obvious that this polynomial interpolation algorithm is more complex than the linear and Sinus Cardinal Interpolation, however it significantly improves system performance especially in the case of a fast fading channel (our case).

On the other hand, the Lagrange Polynomial and the Newton Polynomial interpolation gives the same performances in term of BLER, Throughput and EVM (\%) vs SNR despite the complexity of the Newton method compared to the Lagrange method.

Finally, this algorithm has the advantage of having an adaptable order of polynomial interpolation $\mathrm{n}$ according to conditions of transmission. For example, in our case, we use a channel ITU-Veh A in the Bandwidth of $1.4 \mathrm{MHz}$ where we have $\mathrm{n}=5$ for same Bandwidth but for ITUPEDISTRIAN-B channel $\mathrm{n}=4$.

\section{CONCLUSION}

In the present work, adaptive polynomial interpolation algorithm was described in relation with the method of Lagrange for Downlink LTE system. Simulation is achieved through an ITU-Veh A channel with CQI $=7$ and for MIMO system . We conclude that, despite the complexity of this algorithm (compared to the linear and Sinus Cardinal Interpolation), it offers a considerable improvement of the performance of Downlink LTE system. In effect, using a maximum number of pilots to estimate the parameters of the channel against two for a linear and Sinus Cardinal interpolation optimize considerably the estimation of these parameters. We conclude also, that despite the complexity of Newton method compared to Lagrange method the performances of LTE Downlink system using these two techniques of polynomial interpolation are identical. 
International Journal of Computer Networks \& Communications (IJCNC) Vol.6, No.5, September 2014

Another advantage for our adaptive algorithm, that he offers an adaptive order of polynomial $n$ according to the conditions of system.

\section{REFERENCES}

[1] 3rd Generation Partnership Project, Technical Specification Group Radio Access Network; evolved Universal Terrestrial Radio Access(UTRA): Base Station (BS) radio transmission and reception, pp.22 - 33, TS 36.104, V8.7.0, 2009.

[2] 3rd Generation Partnership Project, Evolved Universal Terrestrial Radio Access (E-UTRA); User Equipment (UE) radio transmission and reception, pp. 22 - 33, ARIB STD-T63-36.101, V8.4.0, 2008.

[3] Nasreddine Mallouki, 'Channel Estimation for LTE Downlink System Based on Lagrange Polynomial Interpolation', mallouki_nasreddine@yahoo.fr, ICWMC, July 2014.

[4] 3rd Generation Partnership Project, Technical Specification Group Radio Access Network; evolved Universal Terrestrial Radio Access (UTRA): Physical Channels and Modulation layer, pp. 55 - 67, TS 36.211, V8.8.0, 2009.S. Caban, Ch. Mehlfuhler, M. Rupp, M. Wriliich, "Evolution of HSDPA and LTE”, Ltd. Published 2012 by John Wiley \&Sons

[5] S. Sesia, I. Toufik, and M. Baker, LTE - The UMTS Long Term Evolution from Theory to Practice, 1st ed, Jonh Wiley and sons, LTD .UK;2009

[6] Technical White paper: "Long Term Evolution (LTE): A Technical Overview,"byMotorola.http://www.motorolasolutions.com/web/Business/Solutions/Industry\%20Solut ions/Service\%20Providers/Wireless\%20Operators/LTE/_Document/Static\%20Files/6834_MotDoc_N ew.pdf

[7]Scott, A.W., Frobenius, Rex, RF Measurements for Cellular Phones and Wireless Data Systems, Wiley/IEEE, 2008

[8] Z. Lin, P. Xiao, B. Vucetic, and M. Sellathurai, "Analysis of receiver algorithms for lte scfdma based uplink systems," IEEE Transaction on Wireless Communications, vol. 9, pp. 60- 65, 2010.

[9] J. F. ValenzuelaValdes, M. A. Garcia Fernandez, A. M. Martinez Gonzalez, and D. A.SanchezHernandez, "Evaluation of true polarization diversity for mimo systems," IEEE Transaction on Antennas and Propagation, vol. 58, pp. 2746-2755, 2009..4

[10] Niru Desai, G. D. Makawana, "Space Diversity for Wireless Communication System- A Issue Review", International Journal of Engineering Science and Innovative Technology (IJESIT) Volume 2, May 2013.University of RENNES internal aggregation UFR MATHEMATICS, (2010).

[11] Technical White paper, "Sampling Theory and Spline Interpolation",Openstax cnx,URL: http://cnx.org/content/ m11126/ latest/

[12] University of RENNES internal aggregation UFR MATHEMATIQUES, (2010) pp. 1 - 5.

[13] 3GPP, TR25.996, V 11.0.0.0," Spatial channel Model for Multiple Input Multiple Output (MIMO)”, 2012

[14] Omar Daoud, Philadelphia University, Jordan PWM Technique to Overcome the Effect of High PAPR in Wireless Systems ,2014

[15] Munjure Mowla, Liton Chandra Paul and Rabiul Hasan, Rajshahi University of Engineering \& Technology, Bangladesh Comparative Performance Analysis of Different Modulation Techniques for PAPR Reduction of OFDM Signal,2014

\section{Authors}

Nasreddine Mallouki, was born in Tunis, Tunisia, on November 1982. From November 2007 until now, he works in National Broadcasting Office, Tunisia. He received the master degree in telecommunication specialty from the National School of Engineering in Tunis (ENIT) in Tunisia in 2011. Currently he is a PhD student at the School of Engineering of Tunis. The research works are realized in Department SYS'COM laboratory in ENIT. His principal research interests lie in the fields of Wireless and Radio Mobile Telecommunications engineering such as MIMO OFDM technology and

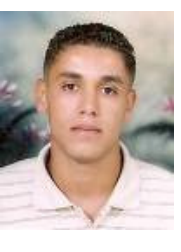
estimation in radio network planning in LTE system. 
Bechir Nsiri, was born in Boussalem, Tunisia, on August 1983. From September 2011 until now, he teaches in Higher Institute of Applied Science and Technology Mateur, Tunisia. He received the master degree in telecommunication specialty from the National School of Engineering in Tunis (ENIT) in Tunisia in 2011. Currently he is a $\mathrm{PhD}$ student at the School of Engineering of Tunis. The research works are realized in Department SYS'COM laboratory in ENIT. His principal research interests lie in the fields of Wireless and Radio Mobile Telecommunications engineering such as MIMO OFDM technology and scheduling in radio network planning in LTE system.

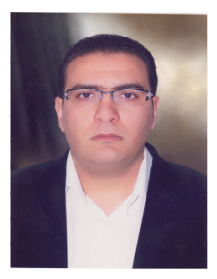

Walid Hakimi is a Regular Professor of Telecommunications at High Institute of technology Study, (Rades, Tunis, Tunisia) since September 1999. From September 2011 until now, he teaches in Electrical Engineering Department, High School of technology and Computing, Tunis, Tunisia. He is a member of SYSCOM laboratory in ENIT. His principal research interests lie in the fields of Wireless and Radio Mobile Telecommunications engineering. He has received the Dipl.-Ing. Degree in electrical engineering from the National school of engineers of Tunis (ENIT), Tunisia. Also, he obtained Doctorat These from University Tunis El Manar, National School of Engineering in Tunis (ENIT), in 2010, in SYS'COM laboratory with collaboration of Telecom Bretagne, Brest, Department SC, CNRS TAMCIC, Technople Brest-Iroise in France.

Mahmoud Ammar was born in Korba, Tunisia. Received the Dipl.- Engi. Degree in electrical engineering from the National School of Engineering in Tunis (ENIT) in Tunisia. He received the M.S. and Doctorat These degrees in telecommunication specialty from Bretagne occidental University in 1999, and 2002, respectively. The research works are realized in Department SC of TELECOM Bretagne, CNRS TAMCIC, Technople Brest-Iroise in France. He is currently working in University Tunis El Manar, National School of Engineering in Tunis (ENIT), Department of

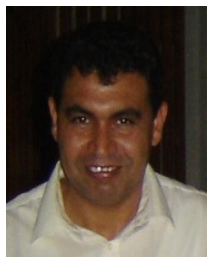
communications and information technologies. Also, he is a member of SYSCOM laboratory in ENIT 